\title{
CHARACTERIZATION OF SURFACE ROUGHNESS AND INITIAL CONDITIONS FOR CYLINDRICAL HYDRODYNAMIC AND MIX EXPERIMENTS
}

\author{
Cris W. Barnes, R. D. Day, N. E. Elliott, \\ S. H. Batha, N. E. Lanier, G. R. Magelssen, J. M. Scott \\ Los Alamos National Laboratory, Los Alamos, NM 87545 \\ (505)665-5687 cbarnes@lanl.gov
}

Steve Rothman, Colin Horsfield, A. M. Dunne, K. W. Parker $A W E$, Aldermaston, Reading RG7 4PR, $U K$

\begin{abstract}
Hydrodynamic experiments in cylindrical geometry are used to study both mix (compressible, in convergent geometry) and mode coupling (impact of short wavelengths on long). For both types of experiments, knowledge of the initial conditions (the surface roughness spectrum, amplitude versus wavelength, as well as all target metrology) is very important. This paper is a discussion of the techniques and efforts to document and understand our initial conditions and their uncertainties and how well we can control them.
\end{abstract}

\section{TARGET DIMENSION MEASUREMENTS}

It is important to measure and document the values as well as the uncertainties in all target dimensions so that one can later analyze the experiment using the target. A detailed description of the fabrication and metrology of the direct drive cylinder targets is provided elsewhere. ${ }^{1}$ There are several dimensional measurements to be made. Densities of ablators, markers, and foams are determined by columnar densitometers with typical $\pm 5 \%$ accuracy. Marker layer thickness for low density, low initial Atwood number, thick dichlorostyrene markers is determined by scanning electron microscope (SEM) images, with uncertainties of $\pm 0.3 \mu \mathrm{m}$; and for thin, high-density gold markers by x-ray fluorescence and/or radiography with $\pm 5 \%$ accuracy. X-ray fluorescence is also used to confirm the spatial extent of embedded markers during processing. Lengths, radii, and thickness of ablators are measured with a laser micrometer during processing. This is done for the value of thickness by making relative difference measurements between the uncoated and coated mandrels (good to $\pm 0.5 \mu \mathrm{m})$. These values are confirmed on a Powell scope or by optical shadowography $( \pm 1.5 \mu \mathrm{m})$ during assembly. Finally, detailed documentation including assembly photographs must be tracked for each target.

\section{SURFACE INTERFACE MEASUREMENTS}

To study the mix arising from shock passage across a variable density interface, that interface must be well characterized. There exist a number of material interfaces in a typical mix experiment such as the direct drive cylinder (DDCYL) targets ${ }^{2,3}$ used at the OMEGA Laser facility $^{4}$ of the Laboratory for Laser Energetics of the University of Rochester. These interfaces include: the central styrene foam surface; both sides of any marker layer, whether high-Z or low; the inside surface of the leached styrene (plastic) ablator; and the outside machined plastic. Figure 1 shows a radial diagram of a typical mix target, illustrating the interfaces of interest. 


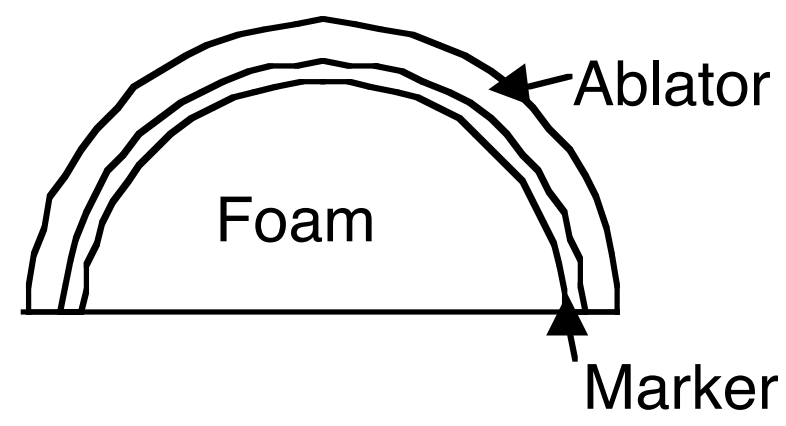

Figure 1: Radial diagram of interfaces in typical cylindrical target.

The physics of interest to be studied and the facility capabilities in laser drive, backlighting, and diagnostics determine the overall target size and marker layer thickness. The growth rates for different wavelengths set, in turn, the wavelengths of most interest. While shorter wavelengths (larger wavenumbers) grow faster and without ablative stabilization at embedded interfaces, as we will show they start smaller. Also, we are interested in comparison of experimental data to Direct Numerical Simulation (DNS) of the turbulence, and initial resolution of the zones of the codes cannot be too small. Thus we are primarily interested in making measurements of the initial surface roughness in the few to a hundred-micron-wavelength range and typically 10 's to perhaps 100's of nm amplitude per mode. Some of our original interest in measuring, characterizing, and affecting the surface roughness was driven by Ablative Rayleigh-Taylor work ${ }^{5}$ and the postulated coupling of short wavelengths (from laser speckle or surface roughness) to longer imposed-perturbation wavelengths of interest. These $10-100 \mu \mathrm{m}$ wavelengths are thus the shortest and fastest growing modes that are not stabilized by ablation.

Tools to characterize the target surfaces include: Scanning Electron Microscope (SEM); Atomic Force Microscope (AFM); Interferometrers (WYKO and ZYGO); and confocal laser scanning microscope. Direct imaging of surfaces with SEM and AFM has been done. Scanning Electron Microscope images of foam surfaces have a relative narrow field of view, cannot see long wavelength variations, but do see "dominant" $\sim 4 \mathrm{~m}$ foam pore size (for $60 \mathrm{mg} / \mathrm{cc} \mathrm{CH}$ foam) (See Figure 2). Such images can be analyzed by turning intensity measurements into a contour map and Fourier analyzing the result. The absolute amplitudes are meaningless, but the relative contributions from different spatial scales (the wavenumber spectrum) can be obtained. Such analysis confirms the same $\mathrm{k}^{-3 / 4}$ scaling seen more quantitatively by interferometric imaging (see

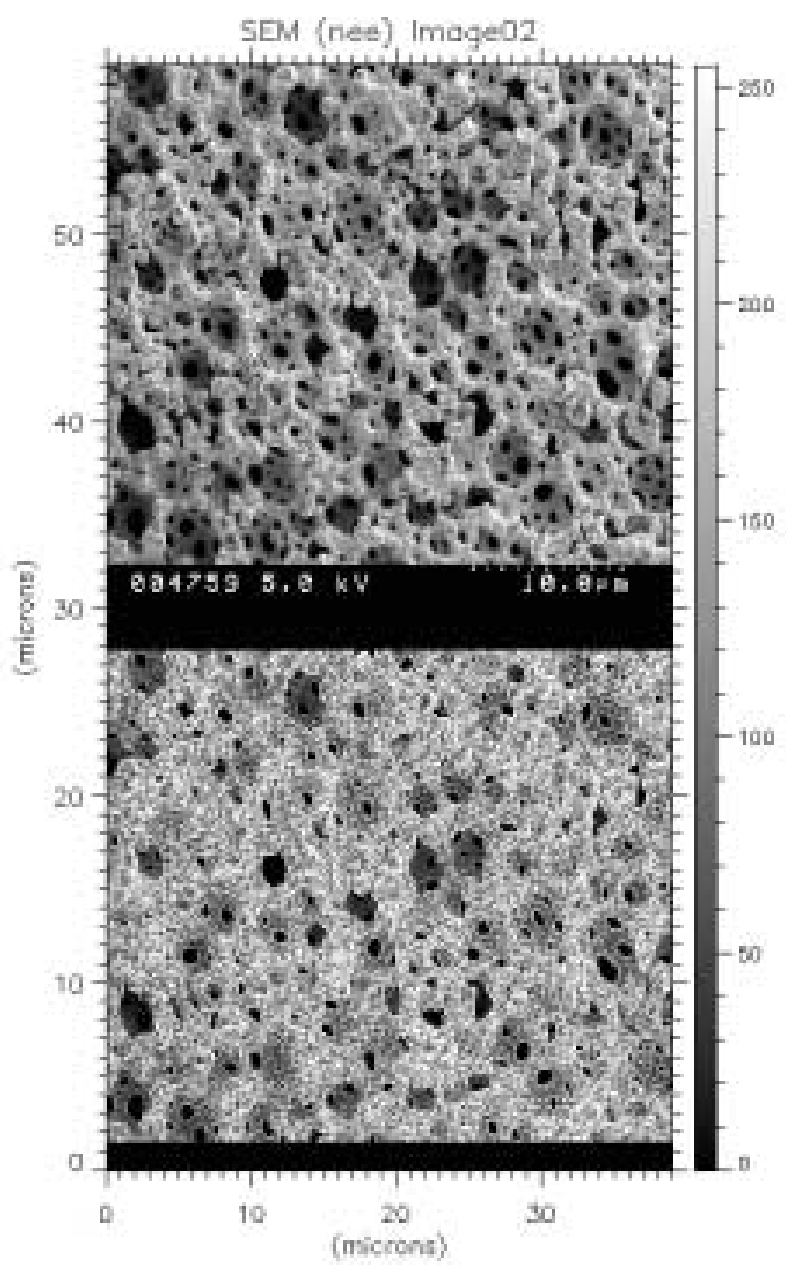

Figure 2: Scanning Electron Microscope (SEM) image of gold vapor-deposited on foam. (Top) SEM image. (Bottom) Contours of intensity of top image.

below) down to at least 1 micron wavelength, and may be flatter after that. The Atomic Force Microscope has a similar limited field of view, limited dynamic range, and other problems that have produced no quantitative information in the spatial scales of interest to us.

\section{INTERFEROMETRIC IMAGING OF SURFACES}

The most quantitative information comes from interferometric imaging of surfaces. Such techniques are well reviewed in a recent reference. ${ }^{6}$ We have used WYKO RTS Plus (at LANL) and ZYGO New View 100 (at AWE) instruments. Comparisons are made between "vertical-scanning (white-light) interferometry" VSI mode and "phase-shifting interferometric" PSI modes. The VSI is best for rough surfaces, but noisier. The PSI mode is less noisy, but has difficulty with strong roughness or 

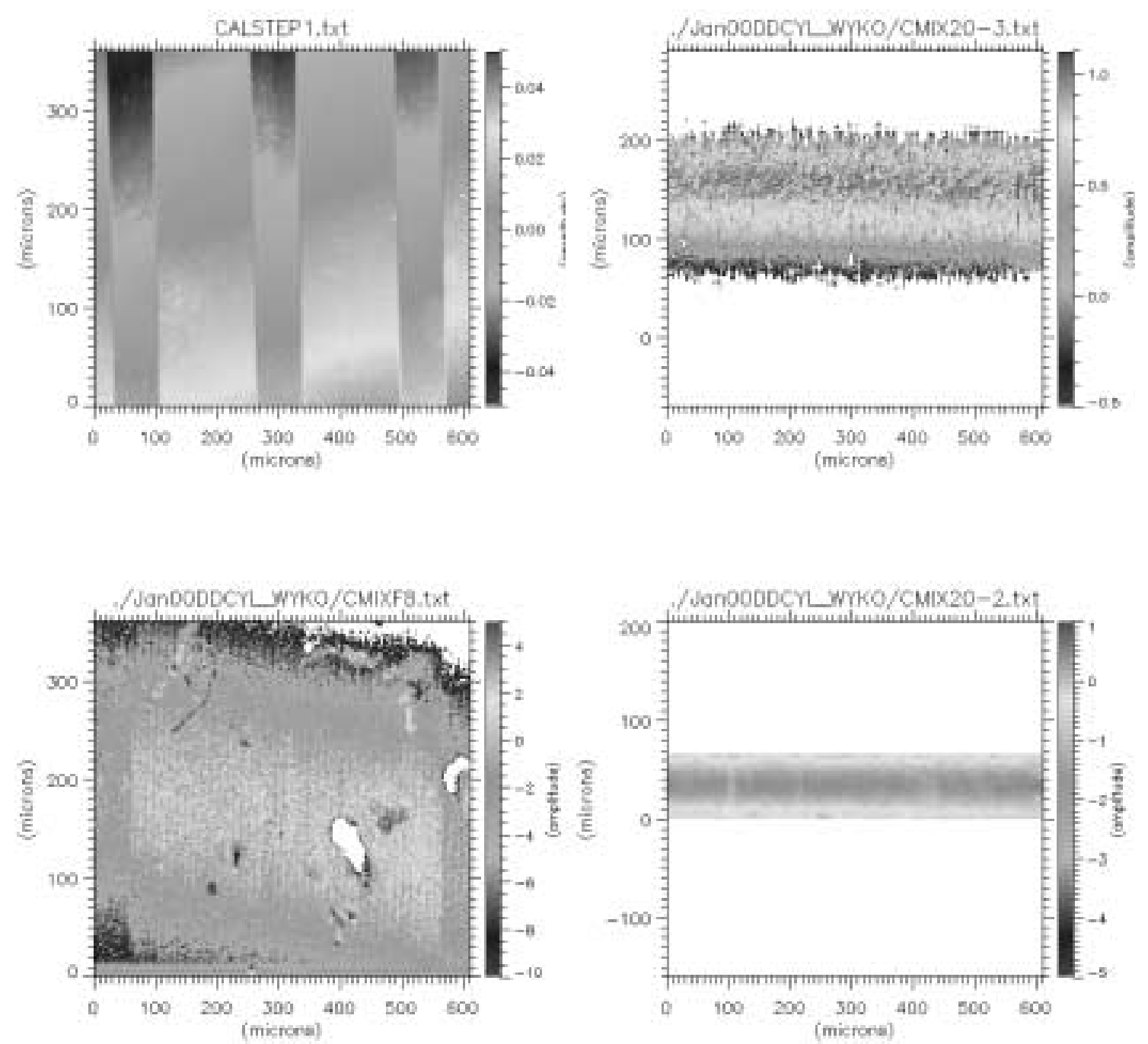

Figure 3: WYKO images of characteristic surfaces. All images are reproduced with the same horizontal and vertical scale. A) NIST-traceable "smooth" flat surface, with 20-nm square-sided grooves, in PSI mode. B) VSI-mode image of gold vapor-deposited on foam. Note thevertical (helical) variation observed. C) VSI mode of outer plastic ablator. D) PSI mode of same plastic ablator.

curvature. For a $860 \mu \mathrm{m}$-diameter cylinder only $50-100 \mu \mathrm{m}$ in the azimuthal direction can be effectively imaged in PSI mode, compared to $300-400 \mu \mathrm{m}$ in the VSI mode. Using a 10X objective allows slightly over $600 \mu \mathrm{m}$ in the axial direction to be viewed in either mode. As the cylinder curves away in azimuth from the point of view of the interferometer, obvious artifacts appear in the data at the edges of the measurement. Electronically masking the measurement area and rejecting the artifacts prevents their inclusion in the data set which would affect overall roughness estimates. The accuracy and uncertainty of these measurements are determined by comparison to standards.
The WYKO interferometer calibration has been checked by comparison to a NIST standard calibration step, and found to be good to 5\% (1 nm out of 20). Figure 3 illustrates typical images of rough, smooth, and calibration surfaces.

The two-dimensional information of the interferometric image is turned into a single surfaceroughness power spectrum. On rough surfaces the interferometer fails to return data from many pixel locations. The data set is interpolated for its amplitude in both the axial and azimuthal directions. This affects the 
estimates of the highest-frequency data but does not change the amplitudes of the primary modes of interest. Twodimensional Fourier transforms can be performed, but the analysis of power in modes of interesting wavelengths is affected by the significant low-mode power caused by curvature in the azimuthal direction. However, the surface roughness still appears isotropic in both azimuthal and axial directions despite the possible differences caused by machining directions. Instead of 2-D Fourier transforms, axial lineouts are used, and after convoluting with a Hanning filter the power spectral density is computed for each one. The power in modes for each axial lineout is then averaged for all azimuthal angles, creating an azimuthallyaveraged spectrum of the axial surface roughness.

For comparison between different instruments, one must take into account the sample length of each image that determines the bandwidth of the Fourier measurement. The spacing between modes in wavenumber space is $2 \pi / \mathrm{L}$ where $\mathrm{L}$ is the sample length. A fair comparison between different instruments is not amplitude versus wavenumber; an instrument with a short sample length will have fewer modes and see more power per mode. Instead the spectral power (the square of the amplitude) divided by the bandwidth (multiplied by $L / 2 \pi$ ) is the correct comparison. Pixel size determines the maximum wavenumber for which information can be determined.

The proof-of-principle mix experiments ${ }^{3}$ compared results from a rough interface of physical-vapor-deposited (PVD) gold-on-foam and from a smoother machined dichlorostyrene layer. Figure 4 repeats surface roughness spectra from the gold surfaces on foam from both optical interferometers and the confocal laser scanning microscope (dashed lines). The power-per-bandwidth drops approximately as a power law with wavenumber to the 1.5 power; that is, the amplitude (the square-root of the power) drops as $\mathrm{k}^{-3 / 4}$. There exist factor-of- 2 variations between samples and measurements. The numerical aperture of the optics appears to make a factor-of-2 difference on rough surfaces. Measurements comparing data on the WYKO from a 10X objective with that from a $40 \mathrm{X}$ objective show approximately such a factor-2 lower amplitude in VSI mode with 10X objective. This helps explain observed differences at this amplitude seen between the LANL and AWE instruments. One also sees what are consistent with helical machining marks on some but not all foam samples (see image in Figure 3b). A combination of the rotation speed on the lathe and the feed rate of the tool could generate these; however, uncertainty in the documentation for these values only confirms the observed wavelength is within an order-of-magnitude of that expected. Also, not every sample shows such a clear single mode. In general, such variations, coupled with inability to characterize every piece

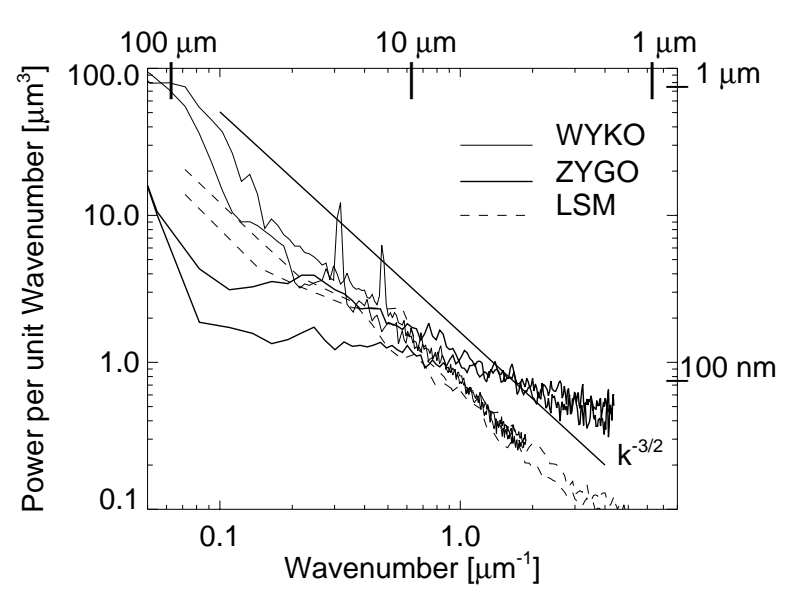

Figure 4: Comparisons of interferometric (WYKO and ZYGO [see text]) and confocal laser scanning microscope measurements of the surface roughness of $60-\mathrm{mg} / \mathrm{cc}$ foam. The data is plotted in power per unit bandwidth to normalize the different sample lengths. Different samples of foam were used which partially explains the different amplitudes seen; in particular, only some samples show the apparent helical machining frequency seen in the WYKO data.

in every location, implies acceptance of a level of uncertainty for matching simulations to experiment of about a factor- 2 in mode amplitude.

The surface roughness can be summarized by a single "average roughness" value, although not particularly accurately. $\mathrm{Ra}$ is the arithmetic average of the absolute value of the surface variation from the mean. Rq is the rms or square root of the mean of the squares of deviations from the mean. These values are very sensitive to the measurements of the longest wavelengths in the sample and may not well reflect difference in surface roughness at the wavelengths of physical interest. That is, long wavelength variations away from the mean may dominate these averages. A better technique is to root-sum-square the spectral amplitude density over some wavelength range and compare surfaces that way. Equivalently in real as opposed to Fourier space, one can look at the variations from the mean over only small sample lengths. The Rsm value $^{6}$ is the mean of the distance between negative-going zero-crossings of the profile trace with its average subtracted. It represents a typical wavelength of the primary features of the profile. 


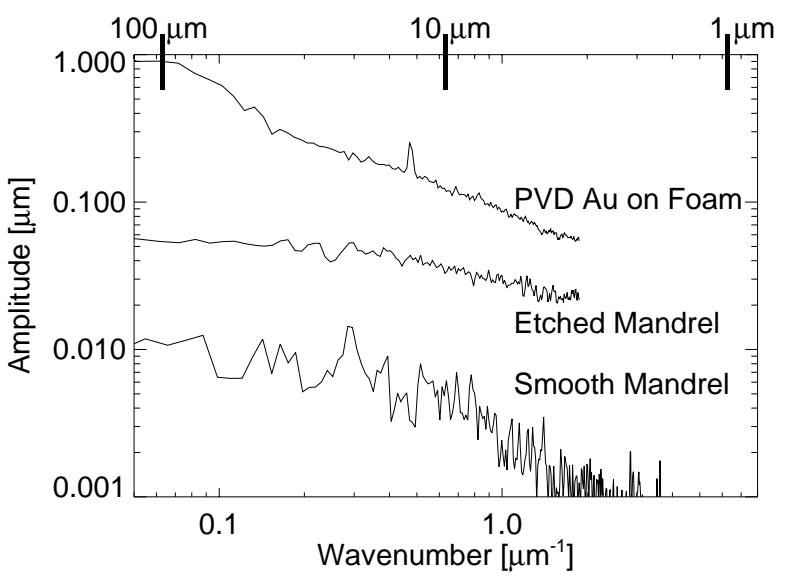

Figure 5: Amplitude vs wavenumber for different goldablator surfaces used in mix experiments. The "PVD foam" and "etched mandrel" spectra use VSI mode; the "smooth" uses PSI mode, and an option to double the number of pixels in the axial view. The pixel size is halved so the sample length is the same and the amplitudes of the spectra are comparable.

\begin{tabular}{l|lll} 
& $\mathrm{Ra}$ & $\mathrm{Rq}$ & $\mathrm{Rsm}$ \\
\hline PVD foam & $1.67 \pm 0.62$ & $2.52 \pm 1.20$ & $20 \pm 7$ \\
Etched & $0.32 \pm 0.03$ & $0.42 \pm 0.06$ & $22 \pm 5$ \\
Smooth & 0.010 & 0.014 & 23
\end{tabular}

All dimensions in microns.

\section{CONTROL OF SURFACE ROUGHNESS}

We are also learning how to affect and control the surface roughness at different interfaces. This is done by: choice of materials and process; different machining techniques; or etching. Marker layers have been created with styrenes by dipping and annealing the polymers. For metal marker layers both physical vapor deposition and electroplating have been used. While those coatings are generally conformal to the surface to which they are applied, they do have different surface characteristics. The machining of the outer surface of interfaces can be varied somewhat by changing the rake angles or feed rates of the diamond tools. If rougher than usual surfaces are desired, use can be made of used bits rather than new bits usually used in the processing. The same process used for leaching the mandrels can be used instead for short periods to etch the surfaces. Dilute solutions of $\mathrm{NaOH}$ for aluminum mandrels and nitric acid for silicon-bronze mandrels are used for the leaching and, where appropriate, for the etching. Dipping the machined mandrels for only a few seconds in the appropriate base or acid roughens them, but also begins to change their radius and make them out of round. The etched mandrels are then electroplated to provide some control over metal markers. This has varied the surface roughness at the pusher-marker interface by over three orders of magnitude. Figure 5 shows the amplitude of the spectral power, and values for overall surface roughness. The value for the smooth case is dominated by slight tilt of the surface causing deviations from the mean; the values quoted are estimates of just the short wavelength deviations. For comparison, the rootsum-square of the amplitudes between 10-50 $\mu \mathrm{m}$ wavelength are $1.53 \mu \mathrm{m}, 0.31 \mu \mathrm{m}$, and $0.05 \mu \mathrm{m}$ for the PVD foam, etched, and smooth cases respectively. We plan to try etching coated mandrels (using $\mathrm{NaOH}$ on aluminum markers put on silicon-bronze mandrels) to further very the obtainable surface roughness. When the layers of the target are completed, the final leaching with the proper dilution also affects the styrene surface, but barely within measurement uncertainty and not systematically in the direction of either smoothing or roughening.

The surface roughness of each interface needs to be tracked during production with multiple measurements. This ability to affect, measure, and characterize surfaces allows us to vary scientifically the initial conditions of our experiment.

\section{THIN DEFLECTION PROBE MEASUREMENTS OF LONG WAVELENGTH FEATURES}

An air-bearing Linear Variable Differential Transformer (LVDT) attached to a very thin deflection probe measures the structure of machined surfaces with initial features at long wavelength. These measurements are limited in spatial resolution and accuracy by the size of the probe tip and the "steepness" of the walls of the features. Calibration steps similar to that used for the WYKO are measured for the thin deflection probes. An example of this type of metrology is the "static targets" used for radiographic image analysis. ${ }^{7}$ Determination of initial amplitudes is important in comparing mode growth to hydro simulations. Figure 6 shows typical data for an $\mathrm{m}=10$ machined perturbation with $1.5-\mu \mathrm{m}$ amplitude used in Nova ablative Rayleigh-Taylor experiments ${ }^{8}$ along with the step calibration data from 10-millionths of an inch $(0.254 \mu \mathrm{m})$ step wedge. 


\section{REFERENCES}
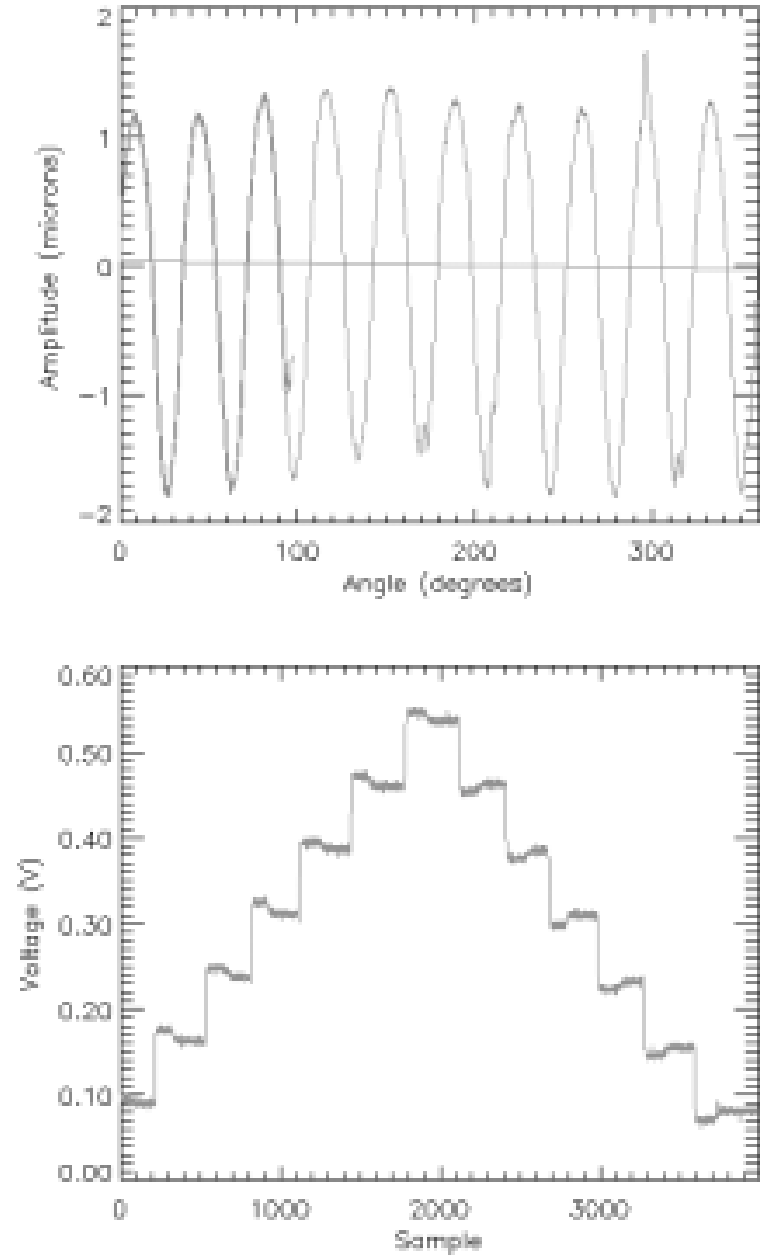

Figure 6: Profilometry of azimuthally sinusoidal perturbations. A) Data for a $\mathrm{m}=10,1.52-\mu \mathrm{m}$ amplitude perturbation. B) Trace of calibration steps used to determine amplitude of data.

\section{ACKNOWLEDGEMENTS}

We acknowledge the technical excellence of Doug Hatch, Gerry Rivera, and David Sandoval in machining and characterizing the long wavelength target features. David Tubbs is thanked for technical leadership of the project and constructive comments on the manuscript. This work is performed under the auspices of the U.S. Department of Energy under contract No. W-7405-Eng-36.

\footnotetext{
${ }^{1}$ N. Elliott et al., "Best Practice Procedures for Making Direct Drive Cylindrical Targets for Studies of Convergent Hydrodynamics," these proceedings.

${ }^{2}$ Cris W. Barnes et al., "Experimental Configuration of Direct Drive Cylindrical Implosions on the OMEGA Laser," Rev. Sci. Instrum. 70 (1999) 471.

${ }^{3}$ Cris W. Barnes et al., "Observation of Mix in a Compressible Plasma in a Convergent Cylindrical Geometry," submitted to Phys. Rev. Letters (2001).

${ }^{4}$ T. R. Boehly et al., "Initial Performance Results of the OMEGA Laser System," Optics Communications 133 (1997) 495.

${ }^{5}$ D. L. Tubbs et al., " Cylindrical Implosion Experiments Using Laser Direct Drive," Phys. Plasmas 6 (1999) 2095.

${ }^{6} \mathrm{~T}$. V Vorburger et al., "Characterization of Surface Topography" in Beam Effects, Surface Topography, and Depth Profiling in Surface Analysis, ed. A W. Czanderna et al., in Methods of Surface Characterization Volume 5, Plenum Press, New York, 1998.

${ }^{7}$ J. M. Scott et al., "Radiographic Image Analysis of Cylindrical Implosion Experiments," Rev. Sci. Instrum. 72 (2001)643.

${ }^{8}$ W. W. Hsing et al., "Rayleigh-Taylor Instability Evolution in Ablatively Driven Cylindrical Implosions," Phys. Plasmas 4 (1997) 1832.
} 